\title{
Empirical antibiotic choice alters microbiological outcomes: Findings from comparative antibiograms in a trauma intensive care unit
}

\author{
S Savage-Reid, ${ }^{1,2}$ MB BCh; M S Moeng, ${ }^{3,4}$ MB BCh, Dip PEC (SA), H Dip Surg (SA), FCS (SA); OrcID 0000-0001-7459-3388; \\ T Thomas ${ }^{1,5}$ MB BCh, MMed (Micro), FCPath Micro (SA), DTM\&H, Dip HIV Man (SA) \\ ${ }^{1}$ Department of Clinical Microbiology and Infectious Diseases, School of Pathology of the University of the Witwatersrand, Johannesburg, South Africa \\ ${ }^{2}$ Clinical Microbiology Laboratory, Charlotte Maxeke Johannesburg Academic Hospital, National Health Laboratory Service, Johannesburg, \\ South Africa \\ ${ }^{3}$ Department of Trauma, University of the Witwatersrand, Johannesburg, South Africa \\ ${ }^{4}$ Milpark Hospital, Johannesburg, South Africa \\ ${ }^{5}$ Infection Control Laboratory, Charlotte Maxeke Johannesburg Academic Hospital, National Health Laboratory Service, Johannesburg, South Africa
}

Corresponding author: S Savage-Reid (estee002@gmail.com)

\begin{abstract}
Background. Inappropriate empirical antibiotics promote antibiotic resistance. Antibiograms guide empirical antibiotic therapy by outlining the percentage susceptibility of each pathogen to individual antibiotics. In 2016, the Trauma Intensive Care Unit at Charlotte Maxeke Johannesburg Academic Hospital escalated empirical antibiotic therapy for nosocomial infections from piperacillin-tazobactam to imipenem plus amikacin. Objectives. This study assessed the impact of escalation in empirical antimicrobial treatment on organism prevalence and resistance profile.

Methods. A retrospective analysis of bacterial and fungal microscopy, culture and susceptibility reports from the laboratory information system of the National Health Laboratory Services, from 1 January 2015 to 31 December 2015 and 1 January 2017 to 31 December 2017, was conducted. Data were de-duplicated according to standard guidelines. Fisher's exact test was used to determine $p$-values.

Results. Organism prevalence shifted between the years, with a $2.7 \%$ increase in streptococci $(p=0.0199), 1.7 \%$ increase in Candida auris $(p=0.0031)$ and $4.6 \%$ and $4.4 \%$ reduction in Acinetobacter baumannii $(p=0.0508)$ and Pseudomonas aeruginosa $(p=0.0196)$, respectively. Similarly, there was a change in the resistance profile, with a $28.9 \%$ reduction in multi-drug resistant (MDR) A. baumannii ( $p=0.0001), 60.4 \%$ reduction in MDR P. aeruginosa $(p=0.0001)$ and a $6.5 \%$ increase in carbapenem-resistant Enterobacterales $(p=0.007)$. The predominant specimen type differed between the years, with significantly more pus, tissue and fluid samples and fewer respiratory samples sent for investigation in 2017 than 2015.

Conclusion. Escalation in the use of empirical antibiotics showed a change in organism prevalence and an improvement in the susceptibility profile of MDR non-fermenters.

Keywords. empirical antibiotics; nosocomial infections; trauma intensive care unit; piperacillin-tazobactam; imipenem; amikacin; antimicrobial resistance.
\end{abstract}

South Afr J Crit Care 2020;36(2):96-103. https://doi.org/10.7196/SAJCC.2020.v36i2.434

Contribution of the study. Current literature on the effects of antibiogram-guided empirical antibiotics is scarce within the South African context. This study shows how antibiograms are an effective antimicrobial stewardship strategy to reduce antimicrobial resistance rates by guiding appropriate choice of empirical antibiotics.

Antimicrobial resistance (AMR) is a threat to public health. In a surveillance report, the World Health Organization (WHO) estimated $87 \%$ of Escherichia coli and $77 \%$ of Klebsiella pneumoniae in the African region were extended-spectrum $\beta$-lactamase (ESBL) producers. In addition, carbapenem-resistant Enterobacterales (CRE) are increasing globally, with reported rates as high as $54 \%$ of all cases involving Enterobacterales. ${ }^{[1]}$ Alarmingly, this rise in AMR is not restricted to the Enterobacterales. Multidrug-resistant (MDR) Gram-negative bacilli such as Acinetobacter baumannii and Pseudomonas aeruginosa are also rising, ${ }^{[2,3]}$ alongside the emergence of MDR Candida auris. ${ }^{[4]}$ Intensive care units (ICUs) are epicentres for AMR because of invasive procedures, prolonged hospitalisations and antibiotic prescriptions in these units. Trauma patients are at further risk of infections because of their breach in host defence systems, surgical interventions, ventilator support, poor nutritional status and medications. ${ }^{[5]}$ Amid this landscape, it is important to ensure appropriate antibiotic usage. Current guidelines advise the 'narrowest-spectrum empirical antibiotic when indicated, to target the most likely pathogen. ${ }^{[6]}$ However, this is rarely practised. In a study assessing outcomes of Staphylococcus aureus bacteraemia, one author found that empirical antibiotics were ineffective in more than a third of patients with methicillin-resistant Staphylococcus aureus (MRSA) blood stream infections. ${ }^{[7]}$ Similarly, a retrospective analysis reported more 
than $50 \%$ of patients with ESBL-producing Enterobacterales. received inadequate empirical antibiotic therapy, with a consequent three-fold increased mortality. ${ }^{[8]}$ For many, the response is to prescribe the broadest spectrum antibiotic while awaiting microbiological results, but this is not without consequence. It is well documented that overuse of antibiotics is driving antibiotic resistance. ${ }^{[9]}$ Conversely, reducing antibiotics can alter resistance. ${ }^{[10]}$ Additionally, inappropriate antibiotic usage causes adverse events in patients and increased costs. Appropriate empirical antibiotic therapy is imperative. One aid in this regard is the antibiogram.

The antibiogram - a summary of microbial pathogens in a unit with percentage susceptibility to possible antimicrobial treatment options (adapted from Merriam Webster Dictionary) - is useful in directing empirical antibiotic choice and tracking resistance trends. Resistance patterns vary between hospitals and often within wards of the same hospital. As a result, antibiograms inform the local epidemiology.

Charlotte Maxeke Johannesburg Academic Hospital (CMJAH), a Johannesburg-based tertiary hospital, receives referrals from many other hospitals throughout Gauteng Province, South Africa (SA), and has several specialised units, such as the trauma ICU. Patients admitted to this unit are adult polytrauma patients, many of whom are critically ill, have prolonged hospitalisation and require several indwelling medical devices (urinary catheters, central lines, drains, etc.) in their management These factors increase their risk of developing infections, particularly with multi-drug resistant organisms (MDROs). ${ }^{[11]}$ In 2016, the trauma ICU in this study changed empirical antibiotic therapy for nosocomial infections from piperacillin-tazobactam to imipenem and amikacin, based on information provided by an antibiogram. This empirical therapy was continued in 2017 based on 6-monthly antibiograms performed in that year. However, the impact of this choice of empirical therapy on the circulating microbes in the ward was unknown. The present study aimed to assess the impact of escalation in empirical antimicrobial treatment on the organism prevalence and resistance profile within the unit.

\section{Methods}

This retrospective, observational analysis of bacterial and fungal identification and susceptibility results used data extracted from the laboratory information system (TrakCare) of the National Health Laboratory
Services (NHLS), from 1 January 2015 to 31 December 2015 and 1 January 2017 to 31 December 2017, to compare organism prevalence, resistance profile and specimen type within the unit, before and after empirical antibiotic escalation. Fig. 1 outlines the procedures and terminology used for this study. All positive microbiological culture reports with antimicrobial susceptibility results for clinical samples sent from the trauma ICU during the study period were included. The following items were excluded:

- screening samples, e.g. rectal and axillary swabs, etc.

- for the antibiogram section, repeat samples showing the same isolated organism for the same patient within 1 year, regardless of body site or resistance profile

- for the specimen type section, repeat blood cultures within 2 weeks, and other repeat samples within 1 month, for the same patient, with the same isolate.

The de-duplication criteria were in keeping with the Clinical and Laboratory Standards Institute (CLSI) guidelines to standardise antibiogram reporting, and the Centers for Disease Control and Prevention surveillance definitions for specific types of infections. ${ }^{[12,13]}$ Antibiograms were constructed for organisms with $>29$ isolates.

During the study period, identification and susceptibility of bacterial and fungal isolates were performed using the automated identification and susceptibility testing system Vitek 2 (BioMérieux, France). From 2017, matrix-assisted laser desorption ionisation time-of-flight mass spectrometry (MALDITOF MS) (Vitek MS BioMérieux, France) was also utilised for identification of some isolates. Isolate identification was reported to species level. Confirmatory susceptibility testing was performed for a subset of isolates, specifically, amikacin disc diffusion for Acinetobacter baumannii, ciprofloxacin disc diffusion for Salmonella spp., and carbapenem e-tests (BioMérieux, France) for CREs. Susceptibility results were only reported for antimicrobials routinely tested by the laboratory, and were interpreted according to the CLSI breakpoints for the 2 years. ${ }^{[14,15]}$ There were no significant changes in the CLSI breakpoints between the years that would affect susceptibility interpretation.

The present study defined MDROs as organisms that were non-susceptible to at

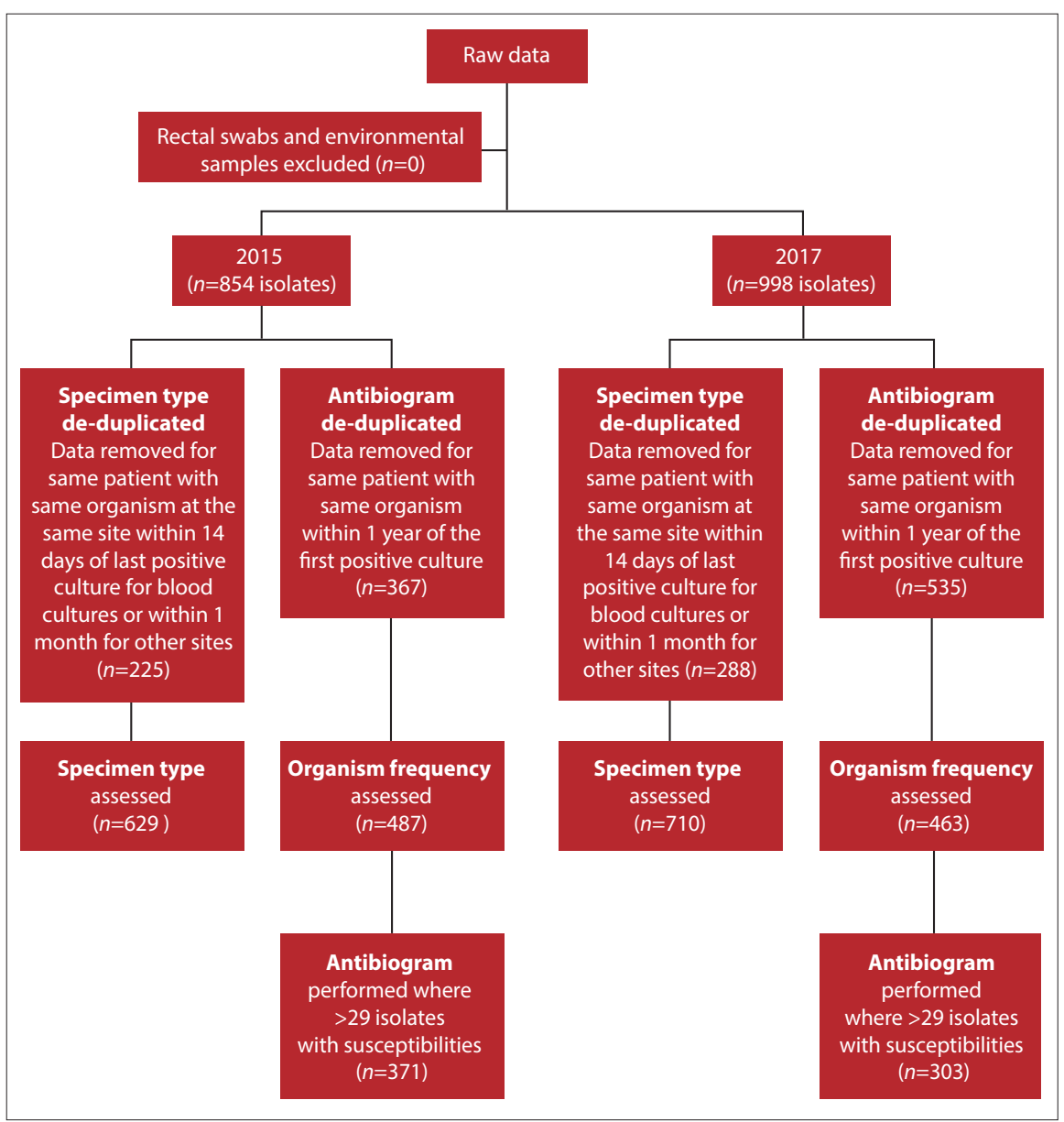

Fig. 1. Data de-duplication process. 
least one agent in $\geq 3$ antimicrobial categories, as per the international standard definitions for acquired resistance. ${ }^{[16]}$ CRE were determined by non-susceptibility to at least one carbapenem on carbapenem e-tests with a positive Hodge or EDTA screening test for confirmation of carbapenemase production. Analysis for extensively-drug resistant (XDR) bacterial organisms (i.e. those susceptible to at least one agent in $\leq 2$ antimicrobial categories) was not possible, as not all agents were routinely tested. Furthermore, colistin susceptibility was not possible as broth microdilution was not performed routinely. Fisher's exact test was used to compare percentages between the two years and to determine $p$-values for statistical significance. A $p$-value of $<0.05$ was considered significant.

\section{Ethics}

Ethics approval was granted by the University of the Witwatersrand's Human Research Ethics Committee (ref. no. M181185).

\section{Results}

A total of 854 reports were extracted in 2015, generating 629 reports for the specimen type section and 487 reports for the antibiogram section, after de-duplication (Fig. 1). In 2017, 998 reports were extracted, with 710 reports for the specimen type and 463 reports for the antibiogram after de-duplication (Fig. 1). In 2017, there were more positive cultures than in 2015, with overall more infections after de-duplication, although the number of organisms after de-duplication was comparable. The key changes in microbiological outcomes between the 2 years are illustrated in Fig. 2.

Table 1 summarises the frequency of organisms isolated in 2015 and 2017. Gram-negative organisms predominated in both years, with $K$. pneumoniae being the most frequently isolated organism, although there was no statistically significant change in the frequency of $K$. pneumoniae $(p>0.05)$. There was an $8.5 \%$ reduction in the number of non-fermenting Gram-negative bacilli isolated ( $p=0.003)$, most notably a $4.6 \%$ reduction in A. baumannii $(p=0.05)$ and a $4.4 \%$ reduction in Pseudomonas aeruginosa $(p=0.019)$. There was no increase with the other non-fermenting Gram-negative bacilli, namely, Stenotrophomonas and Burkholderia. Overall, there were very few Gram-positive organisms, with a $2.7 \%$ increase in all streptococci isolated $(p=0.019)$ in 2017. Similarly, there were very few yeast isolates. However there was a $1.7 \%$ increase in Candida auris infections ( $p=0.003$ ) from 2015 to 2017 .

Only 5 organisms with species-level identification among the Gram-negative organisms had sufficient numbers (>29) to perform an antibiogram. They are depicted in Table 2A. The remaining species with insufficient numbers from the Enterobacterales were grouped together to form an antibiogram. K. pneumoniae susceptibility showed a $13 \%$ increase in amp-C $\beta$-lactamase production $(p=0.0002)$ and a $20.6 \%$ decrease in ESBL producers $(p=0.004)$ in 2017. Conversely, the other Enterobacterales that were grouped together saw a $26.2 \%$ increase in ESBLs $(p=0.0004)$ in 2017. In 2017, a 6.5\% increase in CREs was seen ( $p=0.007$ ), all of which were $K$. pneumoniae.

On analysis of the non-fermenters, A. baumannii showed a significant increase in susceptibility to each class of antibiotic and an overall $28.9 \%$ reduction of MDR A. baumannii ( $p=0.0001$ ). There was a $60.4 \%$ reduction of MDR P. aeruginosa $(p=0.0001)$ in 2017 , although there was no significant change in susceptibility to individual drugs over both years.

Regarding Gram-positive organisms, only S. aureus had sufficient numbers to compile an antibiogram, but this was only possible for 2015 (Table 2B). Of note, the majority of S. aureus isolates (86.7\%) were methicillin-susceptible. The remaining isolates were methicillinresistant and there were no vancomycin-resistant strains.

\section{Specimen type}

Culture-positive specimen type numbers differed between the two years (Table 3). In 2017, there was a significant increase in pus, fluid and tissue samples, and a decrease in respiratory samples occurred when compared with 2015. As seen in Table 4, the majority of the CREs were isolated from fluid or tissue samples. There was no statistically significant change in bloodstream infections, although 7 of the 8 C. auris isolates in 2017 were from blood cultures.

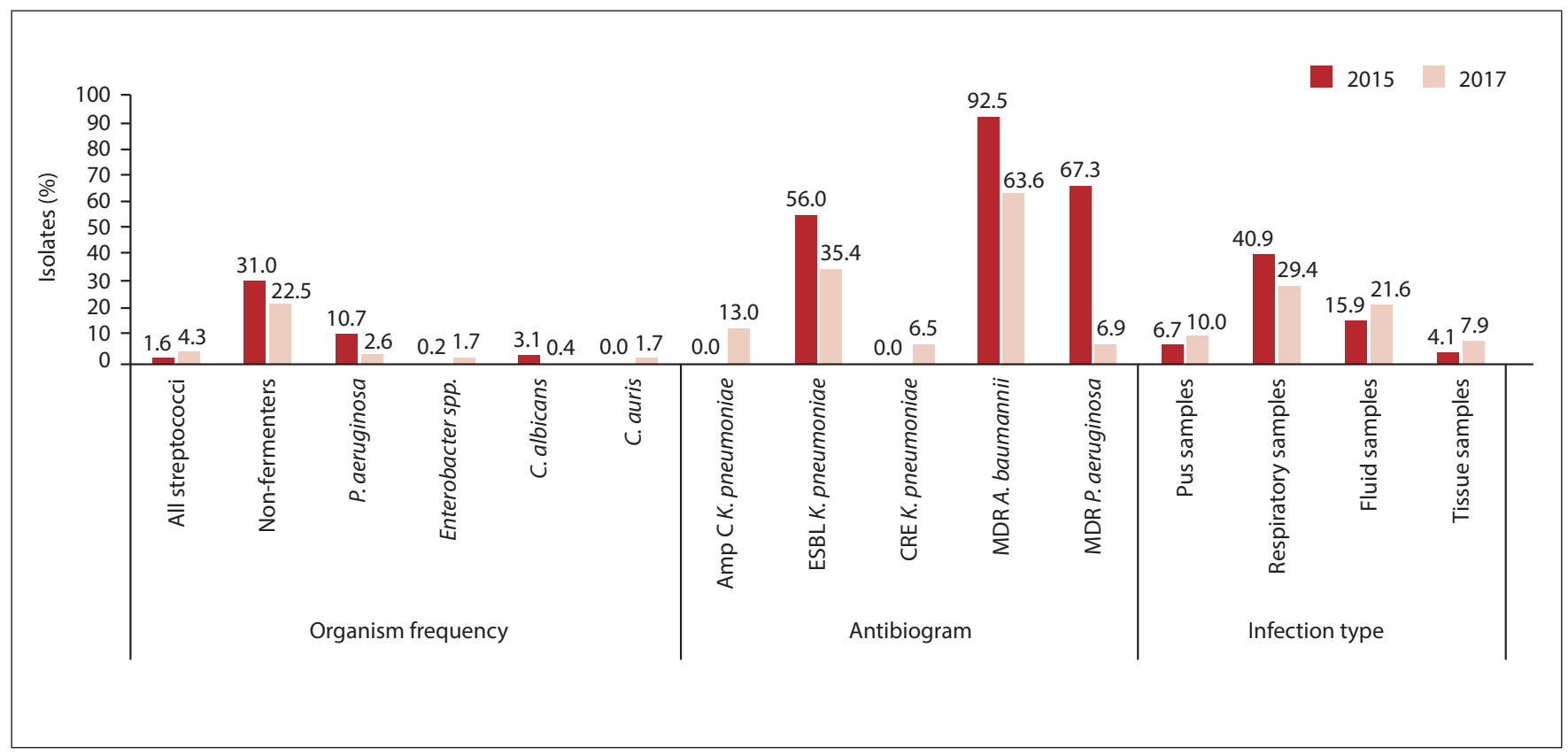

Fig. 2. Summary of key findings. (P. aeruginosa $=$ Pseudomonas aeruginosa; C. albicans $=$ Candida albicans; C. auris $=$ Candida auris; $a m p C=a m p C$ betalactamases; K. pneumoniae $=$ Klebsiella pneumoniae; $E S B L=$ extended-spectrum $\beta$-lactamase, $C R E=$ carbapenem-resistant Enterobacterales; $M D R=$ multidrugresistant; A . baumannii $=$ Acinetobacter baumannii.) 
Table 1. Frequency of isolated organisms

\begin{tabular}{|c|c|c|c|c|c|}
\hline Microorganism & Genus & Species & $2015, n(\%)$ & $2017, n(\%)$ & $p$-value \\
\hline Bacteria, $n(\%)$ & & All & 466 (95.69) & $446(96.33)$ & 0.6244 \\
\hline \multirow[t]{20}{*}{ Gram-positive } & & All & $96(19.71)$ & $111(23.97)$ & 0.1164 \\
\hline & \multirow{3}{*}{ Staphylococcus } & All & $65(13.0)$ & $63(13.61)$ & 0.9245 \\
\hline & & All S. aureus & $30(6.0)$ & $25(5.40)$ & 0.6776 \\
\hline & & All coagulase-negative staphylococci & $35(7.19)$ & $38(8.21)$ & 0.6262 \\
\hline & \multirow[t]{5}{*}{ Enterococcus } & All & $23(2.67)$ & $28(6.05)$ & 0.3902 \\
\hline & & E faecalis & $13(2.67)$ & $17(3.67)$ & 0.4588 \\
\hline & & E. faecium & $10(2.05)$ & $9(1.94)$ & 1.0000 \\
\hline & & E. casseliflavus & 0 & $1(0.22)$ & 0.4874 \\
\hline & & Enterococcus spp. & 0 & $1(0.22)$ & 0.4874 \\
\hline & \multirow[t]{11}{*}{ Streptococcus } & All streptococci & $8(1.64)$ & $20(4.32)$ & 0.0199 \\
\hline & & S. agalactiae & $1(0.21)$ & $0(0)$ & 1.0000 \\
\hline & & S. allolyticus & 0 & $1(0.22)$ & 0.4874 \\
\hline & & S. anginosus & $1(0.21)$ & $4(0.86)$ & 0.2065 \\
\hline & & S. constellatus & 0 & $2(0.43)$ & 0.2373 \\
\hline & & S. dysgalactiae & 0 & $1(0.22)$ & 0.4874 \\
\hline & & S. mitis & $1(0.21)$ & $4(0.86)$ & 0.2065 \\
\hline & & S. pneumoniae & $1(0.21)$ & $5(1.08)$ & 0.1151 \\
\hline & & S. pyogenes & $2(0.41)$ & $1(0.22)$ & 1.0000 \\
\hline & & S. sanguinis & 0 & $1(0.22)$ & 0.4879 \\
\hline & & S. viridans & $2(0.41)$ & $1(0.22)$ & 1.0000 \\
\hline \multirow[t]{30}{*}{ Gram-negatives } & & All & $370(75.96)$ & $335(72.35)$ & 0.2080 \\
\hline & & All non-fermenters & $151(31.00)$ & $104(22.46)$ & 0.0034 \\
\hline & & Acinetobacter baumannii & $80(16.43)$ & $55(11.88)$ & 0.0508 \\
\hline & & A. haemolyticus & 0 & $1(0.22)$ & 0.4874 \\
\hline & & Pseudomonas aeruginosa & $52(10.68)$ & $29(2.63)$ & 0.0196 \\
\hline & & P. putida & $1(0.21)$ & $0(0)$ & 1.0000 \\
\hline & & Burkholderia spp. & $13(2.67)$ & $11(2.38)$ & 0.8380 \\
\hline & & Stenotrophomonas spp. & $5(1.03)$ & $8(1.73)$ & 0.4107 \\
\hline & \multirow[t]{18}{*}{ Enterobacterales } & All & $212(43.53)$ & $221(47.32)$ & 0.2157 \\
\hline & & Citrobacter braakii & $1(0.21)$ & $1(0.22)$ & 1.0000 \\
\hline & & C. freundii & $7(1.44)$ & $7(1.51)$ & 1.0000 \\
\hline & & C. koseri & $3(0.62)$ & $0(0)$ & 0.2497 \\
\hline & & Enterobacter aerogenes & $6(1.23)$ & $8(1.73)$ & 0.5968 \\
\hline & & E. cloacae & $39(8.00)$ & $43(9.29)$ & 0.4906 \\
\hline & & Enterobacter spp. & $1(0.21)$ & $8(1.73)$ & 0.0182 \\
\hline & & Escherichia coli & $30(6.16)$ & $39(8.42)$ & 0.2111 \\
\hline & & E. fergusonii & $1(0.21)$ & $0(0)$ & 1.0000 \\
\hline & & Klebsiella oxytoca & $1(0.21)$ & $1(0.22)$ & 1.0000 \\
\hline & & K. pneumoniae & $85(17.45)$ & $77(1.63)$ & 0.7958 \\
\hline & & Morganella morganii ${ }^{*}$ & $1(0.21)$ & $3(0.65)$ & 0.3620 \\
\hline & & Proteus mirabilis* & $23(4.72)$ & $18(3.89)$ & 0.6324 \\
\hline & & P. penneri ${ }^{*}$ & 0 & $2(0.43)$ & 0.2383 \\
\hline & & P. retgerrii* & $1(0.21)$ & $1(0.22)$ & 1.0000 \\
\hline & & Serratia liquefaciens* & $1(0.21)$ & $1(0.22)$ & 1.0000 \\
\hline & & S. marcescens ${ }^{*}$ & $9(1.85)$ & $10(2.15)$ & 0.8186 \\
\hline & & ${ }^{\star}$ Sum of intrinsically colistin-resistant Enterobacterales & $35(7.19)$ & $35(7.56)$ & 0.9013 \\
\hline & \multirow[t]{4}{*}{ Other Gram-negative organisms } & Haemophilus influenzae & $7(1.44)$ & $8(1.73)$ & 0.7980 \\
\hline & & H. parainfluenzae & 0 & $2(0.43)$ & 0.2373 \\
\hline & & Aeromonas hydrophilia & $3(0.62)$ & $1(0.22)$ & 0.6247 \\
\hline & & A. sobria & 0 & $1(0.02)$ & 0.4879 \\
\hline \multirow[t]{9}{*}{ Yeasts } & & All & $22(4.31)$ & $17(3.67)$ & 0.6244 \\
\hline & & Candida albicans & $15(3.08)$ & $2(0.43)$ & 0.0023 \\
\hline & & C. auris & 0 & $8(1.73)$ & 0.0031 \\
\hline & & C. glabrata & $2(0.41)$ & $3(0.65)$ & 0.6793 \\
\hline & & C. lusitaniae & $0(0)$ & $2(0.43)$ & 0.2373 \\
\hline & & C. parapsilosis & $2(0.41)$ & $1(0.22)$ & 1.0000 \\
\hline & & C. kruseii & $2(0.41)$ & $1(0.22)$ & 1.0000 \\
\hline & & Cryptococcus spp. & $1(0.21)$ & $0(0)$ & 1.0000 \\
\hline & \multicolumn{2}{|l|}{ Total } & $487(100)$ & $463(100)$ & \\
\hline
\end{tabular}


Table 2A. Pooled Gram-negative antibiogram $(n / N, n(\%))$

\begin{tabular}{|c|c|c|c|c|c|c|}
\hline \multirow[b]{3}{*}{ Antibiotic (year) } & \multicolumn{6}{|c|}{ Susceptible isolates/total isolates (\%) } \\
\hline & & & & Other & & \\
\hline & E. cloacae & E. coli & K. pneumoniae & Enterobacterales & A. baumannii & P. aeruginosa \\
\hline \multicolumn{7}{|l|}{ Ampicillin } \\
\hline 2015 & $1 / 39(2.6)$ & $4 / 30(13.3)$ & - & $13 / 44(29.6)$ & - & - \\
\hline 2017 & $1 / 33(3.0)$ & $4 / 39(10.3)$ & - & $14 / 57(24.6)$ & - & - \\
\hline \multicolumn{7}{|c|}{ Amoxicillin-clavulanate } \\
\hline 2015 & $1 / 38(2.6)$ & $19 / 30(63.3)$ & $34 / 85(40.0)$ & $27 / 50(54.0)$ & - & - \\
\hline 2017 & $0 / 43(0.0)$ & $19 / 39(48.7)$ & $30 / 77(39.0)$ & $23 / 60(38.3)$ & - & - \\
\hline \multicolumn{7}{|c|}{ Piperacillin-tazobactam } \\
\hline 2015 & $27 / 39(69.2)$ & $20 / 30(66.7)$ & $60 / 84(71.4)$ & $44 / 50(88.0)$ & $6 / 79(7.6)$ & $38 / 46(82.6)$ \\
\hline 2017 & $22 / 26(84.6)$ & $25 / 33(75.8)$ & $36 / 57(63.1)$ & $41 / 48(85.4)$ & $16 / 49(32.7)^{\dagger}$ & 24/27 (88.9) \\
\hline \multicolumn{7}{|l|}{ Cefoxitin } \\
\hline 2015 & $0 / 39(0.0)$ & $24 / 30(80.0)$ & $85 / 85(100.0)$ & $31 / 55(56.4)$ & - & - \\
\hline 2017 & $0 / 29(0)$ & $27 / 33(81.8)$ & $47 / 54(87.0)^{\dagger}$ & $20 / 50(80.0)$ & - & - \\
\hline \multicolumn{7}{|l|}{ Ceftriaxone } \\
\hline 2015 & $26 / 37(70.3)$ & $18 / 30(60.0)$ & $37 / 84(44.0)$ & $51 / 55(92.7)$ & - & - \\
\hline 2017 & $23 / 29(79.3)$ & $22 / 33(66.7)$ & $34 / 56(60.7)$ & $33 / 52(63.5)^{*}$ & - & - \\
\hline \multicolumn{7}{|l|}{ Ceftazidime } \\
\hline 2015 & $30 / 39$ (76.9) & $19 / 30(63.3)$ & $41 / 85(48.2)$ & $50 / 55(90.0)$ & 7/80 (8.8) & $41 / 52(78.9)$ \\
\hline 2017 & $25 / 30(83.3)$ & $24 / 34(70.6)$ & $35 / 57(61.4)^{\varsigma}$ & $39 / 51(76.5)$ & $21 / 51(41.2)^{\ddagger}$ & 24/27 (88.9) \\
\hline \multicolumn{7}{|l|}{ Cefepime } \\
\hline 2015 & $30 / 39(76.9)$ & $20 / 30(66.7)$ & $42 / 85(49.4)$ & $52 / 55(94.6)$ & $6 / 80(7.5)$ & $38 / 52(73.1)$ \\
\hline 2017 & $25 / 30(83.3)$ & $25 / 32(78.1)$ & $34 / 56(60.7)$ & $37 / 48(77.1)^{*}$ & $19 / 51(37.3)^{\ddagger}$ & $24 / 27(88.9)$ \\
\hline \multicolumn{7}{|l|}{ Ertapenem } \\
\hline 2015 & $39 / 39(100)$ & $30 / 30(100)$ & $85 / 85(100)$ & $55 / 55(100)$ & - & - \\
\hline 2017 & $27 / 27(100)$ & $33 / 33(100)$ & $47 / 52(90.4)$ & $48 / 48(100)$ & - & - \\
\hline \multicolumn{7}{|l|}{ Imipenem } \\
\hline 2015 & $39 / 39(100)$ & $30 / 30(100)$ & $85 / 85(100)$ & $37 / 37(100)$ & $6 / 80(7.5)$ & $37 / 52(71.2)$ \\
\hline 2017 & $29 / 29(100)$ & $33 / 33(100)$ & $56 / 56(100)$ & $46 / 46(100)$ & $17 / 49(34.7)^{\S}$ & $24 / 27(88.9)$ \\
\hline \multicolumn{7}{|l|}{ Meropenem } \\
\hline 2015 & $39 / 39(100)$ & $30 / 30(100)$ & $85 / 85(100)$ & $55 / 55(100)$ & $6 / 80(7.5)$ & $39 / 52(75.0)$ \\
\hline 2017 & $31 / 31(100)$ & $34 / 34(100)$ & $59 / 59(100)$ & $49 / 49(100)$ & $20 / 51(39.2)^{\ddagger}$ & $25 / 28(89.3)$ \\
\hline \multicolumn{7}{|l|}{ Ciprofloxacin } \\
\hline 2015 & $38 / 39(97.4)$ & $22 / 29(75.9)$ & $76 / 85(89.4)$ & $54 / 55(98.2)$ & $33 / 79$ (41.8) & $46 / 52(88.5)$ \\
\hline 2017 & $22 / 27(81.5)$ & $19 / 22(86.4)$ & $35 / 45(77.8)$ & $26 / 30(86.7)$ & $39 / 53(73.6)^{\varsigma}$ & $27 / 29(93.1)$ \\
\hline \multicolumn{7}{|l|}{ Gentamicin } \\
\hline 2015 & $28 / 38(73.7)$ & $28 / 30(93.3)$ & $41 / 85(48.2)$ & $52 / 55(94.6)$ & $25 / 79$ (31.6) & $45 / 52(86.5)$ \\
\hline 2017 & $30 / 36(83.3)$ & $27 / 36(75.0)$ & $33 / 65(50.8)$ & $47 / 51(92.2)$ & $33 / 55(60.0)^{*}$ & $24 / 29(82.8)$ \\
\hline \multicolumn{7}{|l|}{ Amikacin } \\
\hline 2015 & $34 / 39(87.2)$ & $30 / 30(100)$ & $79 / 84(94.1)$ & $52 / 52(100)$ & $13 / 24(54.2)$ & $46 / 51(90.2)$ \\
\hline 2017 & $41 / 43(95.4)$ & $38 / 38(100)$ & $67 / 77(87.0)$ & $60 / 60)(100)$ & $34 / 50(68)$ & $39 / 41(95.1)$ \\
\hline Resistance profile & & & & lates & & \\
\hline \multicolumn{7}{|l|}{$\mathrm{AmpC}$} \\
\hline 2015 & 100 & 20.0 & 0 & 43.6 & - & - \\
\hline 2017 & 100 & 18.2 & $13.0^{\varsigma}$ & $20.0^{\S}$ & - & - \\
\hline \multicolumn{7}{|l|}{ ESBL } \\
\hline 2015 & 23.1 & 40.0 & 56.0 & 10.3 & - & - \\
\hline 2017 & 16.7 & 33.3 & $35.4^{*}$ & $36.5^{\ddagger}$ & - & - \\
\hline \multicolumn{7}{|l|}{ CRE } \\
\hline 2015 & 0 & 0 & 0 & 0 & - & -- \\
\hline 2017 & 0 & 0 & $6.49^{*}$ & 0 & - & \\
\hline MDR & & & & & & \\
\hline 2015 & - & - & - & - & 92.50 & 67.31 \\
\hline 2017 & - & - & - & - & $63.64^{\ddagger}$ & $6.89^{*}$ \\
\hline $\begin{array}{l}\text { E. cloacae }=\text { Enterobacter } \\
\text { CRE }=\text { carbapenem-resi } \\
{ }^{*} p \leq 0.05 . \\
\vdots p \leq 0.001 . \\
+p \leq 0.0001 . \\
p p \leq 0.0005 .\end{array}$ & $\begin{array}{l}\text { = Escherichia ce } \\
\text { terales; } \mathrm{MDR}=\end{array}$ & $\begin{array}{l}\text { umoniae }=\text { Kleb } \\
\text { g-resistant; - }\end{array}$ & $\begin{array}{l}\text { reumoniae; } \mathrm{AmpC}= \\
\text { icable. }\end{array}$ & $\mathrm{C}$ beta-lactamases; ESI & $=$ extended spectr & ctamase; \\
\hline
\end{tabular}




\section{Discussion}

The present study assessed the impact of escalation in empirical antimicrobial treatment from piperacillin-tazobactam to imipenem and amikacin on organism prevalence and resistance profile within the trauma ICU at CMJAH. This escalation saw two main changes: (i) a decrease in the prevalence of non-fermenting Gram-negative bacilli, most notably in A. baumannii; and P. aeruginosa, (ii) accompanied by a reduction in MDR isolates among these organisms. These findings highlight the impact of empirical antibiotic choice on the organism prevalence and resistance profile.

Table 2B. Antibiogram for Staphylococcus aureus (2015)

\begin{tabular}{ll}
\hline Antibiotic (number of isolates tested) & Susceptible isolates, $\boldsymbol{n}$ (\%) \\
\hline MSSA (30) & $26(86.67)$ \\
Penicillin/ampicillin & - \\
Ceftaroline (0) & - \\
Vancomycin (29) & $29(100)$ \\
Cefoxitin (30) & $26(86.67)$ \\
Teicoplanin(25) & $25(100)$ \\
Clindamycin(30) & $25(83.33)$ \\
Gentamycin (29) & $27(93.10)$ \\
Ciprofloxacin (30) & $25(83.33)$ \\
Co-trimoxazole (30) & $23(76.67)$ \\
Fusidic acid (0) & - \\
Tetracyclines (26) & $19(73.08)$ \\
Fosfomycin (0) & - \\
Tigecycline (25) & $25(100)$ \\
Chloramphenicol (2) & $2(100)$ \\
Quinopristin-dalfopristin (0) & - \\
Linezolid (30) & $30(100)$ \\
Erythromycin (30) & $25(83.33)$ \\
MSSA = methicillin-susceptible Staphylococcus aureus.
\end{tabular}

Table 3. Comparison of culture-positive specimen types in 2015 v. 2017

\begin{tabular}{|c|c|c|c|}
\hline Specimen type & $2015, n(\%)^{\star}$ & $2017, n(\%)^{*}$ & $p$-value \\
\hline Bood culture & $151(24.00)$ & $166(23.38)$ & 0.7970 \\
\hline Cerebrospinal fluid & $1(0.16)$ & $1(0.14)$ & 1.0000 \\
\hline Central line & $1(0.16)$ & 0 & 0.47 \\
\hline Pus & $42(6.68)$ & $71(10.00)$ & 0.03 \\
\hline Respiratory & $257(40.86)$ & $209(29.44)$ & 0.0001 \\
\hline Fluid & $100(15.89)$ & $153(21.55)$ & 0.009 \\
\hline Tissue & $26(4.13)$ & $56(7.89)$ & 0.004 \\
\hline Urine & $51(8.11)$ & $54(7.61)$ & 0.76 \\
\hline All $(N)$ & 629 & 710 & \\
\hline
\end{tabular}

The decrease in prevalence and resistance rates of A. baumannii and $P$. aeruginos $a$ is promising. Non-fermenting Gram-negative bacilli can cause life-threatening illnesses in critically ill patients and are intrinsically resistant to many antibiotics, limiting treatment options. Among these limited antibiotic choices, further resistance is developing. ${ }^{[17]}$ These organisms are notorious for colonising patients and hospital environmental surfaces, causing nosocomial infections and the spread of antimicrobial resistance to other bacteria through horizontal gene transfer. ${ }^{[18]}$ The findings of this study suggest that empirical antibiotic choice has an important role in affecting the microbiological epidemiology within a unit, particularly of non-fermenting Gram-negative bacilli, which could alter the numbers of nosocomial infections.

Other studies show conflicting results on the effect of empirical antibiotics on organism prevalence and resistance. A retrospective review in a neonatal ICU showed that switching from cefotaxime to tobramycin reduced ESBL infection rates. ${ }^{[19]}$ Similarly, a prospective study in a medical ICU in Italy showed a reduction in MRSA and piperacillin-tazobactam-resistant $P$. aeruginosa when empirical antibiotics were changed from piperacillin-tazobactam to imipenem for nosocomial pneumonias. ${ }^{[20]}$ In contrast, a recent multi-centre randomised control trial showed that antibiotic cycling did not reduce carriage of Gram-negative bacteria in ICU patients. ${ }^{[21]}$ However, this trial used 6-weekly periods of cycling, which was a much shorter period than in our study. In light of these conflicting results, more studies assessing longer periods are needed to ascertain the effect of empirical antibiotics on resistance rates.

The frequency of organisms isolated in both years was similar to those seen in other ICUs. In an international point-prevalence study spanning 75 countries, Gram-negative organisms were the most frequently isolated organisms from microbial cultures, followed by Gram-positive organisms and then fungi. ${ }^{[22]}$ At a local level, similar findings are noted. In a study assessing cumulative antibiograms over two periods in an adult medical ICU and high care at CMJAH, Law et al. ${ }^{[23]}$ documented a predominance of Enterobacterales. Furthermore, in a trauma ICU at Inkosi Albert Luthuli Central Hospital, Ramsamy et al. ${ }^{[2]}$ describe a similar microbiological epidemiological pattern, with Gram-negative pathogens predominating, followed by Gram-positive pathogens and very few fungi. However, this trend was not seen in a 5-year review of data from the same province, in which S. aureus was the most frequently isolated pathogen. Similarly, von Knorring et al. ${ }^{[25]}$ analysed cumulative antibiograms for a paediatric oncology unit at CMJAH and showed comparatively higher rates of Gram-positive isolates and yeasts, ${ }^{[26]}$ which would alter empirical antibiotic choice. This stresses the value of unit-specific antibiograms in guiding empirical antibiotics, as resistance patterns vary between wards, even within the same hospital. One study comparing antibiotic susceptibilities within a hospital and a surgical

Table 4. Specimen types for organisms with significant changes between 2015 and 2017 (n)

\begin{tabular}{|c|c|c|c|c|c|c|c|c|c|c|c|c|}
\hline \multirow[b]{2}{*}{ Microorganism } & \multicolumn{2}{|c|}{ Blood culture } & \multicolumn{2}{|c|}{ Urine } & \multicolumn{2}{|c|}{ Respiratory } & \multicolumn{2}{|c|}{ Fluid } & \multicolumn{2}{|c|}{ Tissue } & \multicolumn{2}{|c|}{ Pus } \\
\hline & 2015 & 2017 & 2015 & 2017 & 2015 & 2017 & 2015 & 2017 & 2015 & 2017 & 2015 & 2017 \\
\hline CRE & 0 & 0 & 0 & 0 & 0 & 1 & 0 & 3 & 0 & 1 & 0 & 0 \\
\hline C. auris & 0 & 7 & 0 & 1 & 0 & 0 & 0 & 0 & 0 & 0 & 0 & 0 \\
\hline All streptococci & 3 & 6 & 0 & 0 & 1 & 5 & 2 & 5 & 1 & 2 & 1 & 2 \\
\hline A. baumannii & 18 & 17 & 7 & 9 & 63 & 55 & 8 & 19 & 3 & 1 & 4 & 3 \\
\hline$P$. aeruginosa & 8 & 8 & 3 & 4 & 45 & 31 & 12 & 8 & 2 & 3 & 5 & 10 \\
\hline Total & 29 & 38 & 10 & 14 & 109 & 92 & 22 & 35 & 6 & 7 & 10 & 15 \\
\hline
\end{tabular}


ICU found significant differences between the two, suggesting that unitspecific antibiograms could better guide empirical treatment. ${ }^{[27]}$

The increase in cultures for certain specimen types (pus, fluid and tissue) and decrease in others (respiratory samples) between the 2 years is difficult to interpret. While this could be due to pharmacokinetic properties of individual drugs resulting in different levels of drug penetration at different body sites, other factors could play a role, namely culturing practices or changes in clinical presentation. Further studies that assess clinical outcomes are needed to ascertain whether changes in empirical antibiotics alter infection site.

Of note, most $A$. baumannii and $P$. aeruginosa in both years were cultured from respiratory samples, including sputum samples. The isolates may represent colonisation rather than infection. However, colonisation precedes infection, and for this reason, although the present study is unable to distinguish colonisation from infection, the reduction in non-fermenting Gram-negative bacilli is still significant.

The findings of this study show that microbiological surveillance is fundamental to guiding empirical antibiotic choice as a stewardship strategy. This fact was also highlighted in a local study by Ramsamy et al.: ${ }^{[23]}$ when combined with active surveillance, empirical antibiotic policies were adequate in more than $90 \%$ of cases, thus reducing the need for ultrabroad-spectrum antibiotics. The authors emphasise that empirical antibiotics exert pressure on the microbial environment and contribute towards total antibiotic use. Coupled with a delay in obtaining culture results and failure to de-escalate therapy, inappropriate empirical antibiotics contribute significantly to AMR and provide an opportune target for AMS strategies.

\section{Study strengths and limitations}

The present study has numerous strengths. The international guidelines and standardised definitions used to develop the antibiogram and to define MDROs facilitate comparison with other studies. Another strength is that analysis was not restricted to resistance profiles alone, but included specimen type and organism prevalence, allowing for detection of changes in specimen type, spectrum of organisms and resistance profile, all of which are important considerations when selecting empirical antibiotics.

Nevertheless, a number of limitations exist. Firstly, since this was a retrospective observational analysis of laboratory data, correlation with clinical outcomes was not assessed, and for this reason, infection site was not assessed. Additionally, antibiotic susceptibility testing was not performed uniformly on all isolates. This may have been due to logistical reasons, where certain antimicrobial susceptibilities were missed if not deemed clinically necessary. These antibiotics included fosfomycin, chloramphenicol and tobramycin. Furthermore, only antibiotic agents routinely tested in the diagnostic microbiology laboratory were available for analysis. As a result, certain antibiotics were not tested for the Gram-negative organisms, namely ceftaroline, doripenem, aztreonam, ampicillin-sulbactam, colistin and doxycycline. For these reasons, XDR organisms could not be analysed. XDR organisms are defined based on susceptibility to at least one antibiotic agent in two or less antimicrobial categories. ${ }^{[16]}$ This definition requires that all categories of antibiotics are tested and that every agent in each category is tested.

Furthermore, relying solely on categorical interpretation of isolates as either resistant or susceptible introduces several limitations. Firstly, there is an increasing recognition of the importance of minimum inhibitory concentration (MIC)-guided antibiotic therapy ${ }^{[28]}$ Relying on categorical interpretations alone will miss subtle creeps in the MIC, which can affect patient outcomes. In addition, resistance could have been overcalled and higher antibiotic doses could have been used to treat the 'resistant' isolate. For instance, CLSI has a 'susceptible dosedependent' for the cefepime use in the Enterobacterales. This is an MIC range between the susceptible and intermediate categories. Isolates that fall into this range can be treated with cefepime, provided that higher dosing strategies are used. ${ }^{[14,15]}$ However, categorical interpretation of antibiograms would falsely categorise these isolates as resistant to cefepime. Lastly, it is important to assess MICs in relation to sitespecific penetration of antibiotics, considering that a blood stream infection caused by an uncomplicated urinary tract infection could be treated with certain agents that would be inadequate for a blood stream infection in a patient with tertiary peritonitis. This is particularly relevant since trauma patients are at high risk of complicated intraabdominal infections or ventilator-associated pneumonias which can pose difficulties to antibiotic penetration.

Lastly, the retrospective nature of this study prevents any immediate intervention based on the findings being instituted. However, it highlights the fact that yearly antibiogram comparisons and re-evaluation of empirical therapy will improve the infection profile in hospital units.

There are several barriers to extrapolating these findings to other units. Firstly, there is the issue of differing reporting. While national surveillance uses the number of isolates cultured as the denominator to determine percentage susceptibility, the WHO recommends using admission numbers and days of hospitalisation for intra- and interhospital comparison. ${ }^{[29]}$ This issue highlights the need for uniform reporting of AMR. Furthermore, patient risk profile, clinician culturing practices and infection control compliance differ between units, and direct comparisons would not assess these confounding factors. ${ }^{[25]}$ However, although direct comparisons cannot be made between units regarding organism prevalence and resistance rates, the findings of this study underline the potential benefit of antibiogram-guided empirical therapy in any unit. A recent review of antimicrobial stewardship in SA outlines particular challenges to its implementation (paucity of data, limited healthcare facility infrastructure, staff shortages, laboratory inaccessibility and lack of quality-assured antibiotics), and stresses the need to maximise gains from 'low-hanging fruit', which are easy to implement. ${ }^{[30]}$ Amid a climate of growing antimicrobial resistance and limited treatment options, antimicrobial stewardship has become crucial, and antibiograms provide an invaluable surveillance tool to guide empirical antibiotic choice in this regard.

\section{Conclusion}

This study shows a change in organism prevalence and susceptibility profiles, along with changes in specimen type numbers over the 2 years, with the escalation in empirical antibiotic therapy from piperacillintazobactam to imipenem-amikacin combination therapy. These findings emphasise the value of cumulative antibiograms in guiding empirical antimicrobial choice, and underline their importance in preventing antimicrobial resistance.

Declaration. This manuscript was submitted in partial fulfilment of an MMed (Microbiology)

Acknowledgements. The authors thank Mr Naseem Cassim for his help with data de-duplication and statistical analysis.

Author contributions. SSR: contributed to study design, performed data analysis; write-up of manuscript. MSM: contributed to study design; participated in manuscript revisions. TT: conceptualisation of study; contributed to study design; critically reviewed data interpretation and scientific content of paper; approved final version for publication. 


\section{Funding. None.}

\section{Conflicts of interest. None.}

1. World Health Organization. Antimicrobial Resistance: Global Report on Surveillance. Geneva: WHO, 2014. https://apps.who.int/iris/handle/10665/112642 (accessed 31 January 2020).

2. Gniadek TJ, Carroll KC, Simner PJ. Carbapenem-resistant non-glucose-fermenting Gramnegative bacilli: The missing piece to the puzzle. J Clin Microbiol 2016;54(7):1700-1710. https:// doi.org/10.1128/jcm.03264-15

3. Osei SJ. Current state of resistance to antibiotics of last resort in South Africa: A review from a public health perspective. Front Public Health 2016;4:209. https://doi.org/10.3389/ fpubh.2016.00209

4. Jeffery-Smith A, Taori SK, Schelenz S, et al. Candida auris: A review of the literature. Clin Microbiol Rev 2017;31(1):00029-00170. https://doi.org/10.1128/cmr.00029-17

5. Morgan AS. Risk factors for infection in the trauma patient. J Natl Med Assoc 1992;84:1019-1023.

6. Wasserman S, Boyles T, Mendelson M. Pocket guide to antibiotic prescribing for adults in South Africa, 2015. https://www.fidssa.co.za/Content/Documents/SAASP_Antibiotic_Guidelines_2015. pdf (accessed 31 January 2020).

7. Bassetti M, Trecarichi EM, Mesini A, et al. Risk factors and mortality of healthcare-associated and community-acquired Staphylococcus aureus bacteraemia. Clin Microbiol Infec 2012;18(9):862869. https://doi.org/10.1111/j.1469-0691.2011.03679.x

8. Tumbarello M, Sanguinetti M, Montuori E, et al. Predictors of mortality in patients with bloodstream infections caused by extended-spectrum B-lactamase-producing Enterobacteriaceae: Importance of inadequate initial antimicrobial treatment. Antimicrob Agents Chemother 2007;51(6):1987-1994. https://doi.org/10.1128/aac.01509-06

9. Shallcross LJ, Davies DSC. Antibiotic overuse: A key driver of antimicrobial resistance. Br J General Pract 2014;64(629):604-605. https://doi.org/10.3399/bjgp14x682561

10. Friedman CR, Whitney CG. It's time for a change in practice: Reducing antibiotic use can alter antibiotic resistance. J Infect Dis 2008;197(8):1082-1083. https://doi.org/10.1086/533450

11. Brusselaers N, Vogelaers D, Blot S. The rising problem of antimicrobial resistance in the intensive care unit. Ann Intensive Care 2011;1(1):47. https://doi.org/10.1186/2110-5820-1-47

12. Clinical and Laboratory Standards Institute. Analysis and presentation of cumulative antibiotic susceptibility test data: Approved guideline 2014. Wayne: CLSI, 2014. https://clsi.org/standards/ products/microbiology/documents/m39/ (accessed 31 January 2020).

13. Centers for Disease Control and Prevention/National Health Safety Network. CDC/NHSN Surveillance Definitions for Specific Types of Infections 2019. https://www.cdc.gov/nhsn/pdfs/ pscmanual/17pscnosinfdef_current.pdf (accessed 31 January 2020).

14. Clinical and Laboratory Standards Institute. Performance Standards for Antimicrobial Susceptibility Testing. 25th ed. Wayne, PA: CLSI, 2015.

15. Clinical and Laboratory Standards Institute. Performance Standards for Antimicrobial Susceptibility Testing. Wayne, PA: CLSI, 2017.

16. Magiorakos A-P, Srinivasan A, Carey RB, et al. Multidrug-resistant, extensively drug-resistant and pandrug-resistant bacteria: An international expert proposal for interim standard definitions for acquired resistance. Clin Microbiol Infec 2012;18(3):268-281. https://doi.org/10.1111/j.14690691.2011.03570.x
17. Paterson DL, Bonomo RA. Multidrug-resistant Gram-negative pathogens: The urgent need for 'old' polymyxins. Polymyxin antibiotics: From laboratory bench to bedside. Adv Exp Med Bio 2019;1145:9-13. https://doi.org/10.1007/978-3-030-16373-0_2

18. Mukerii S, O'Dea M, Barton M, et al. Development and transmission of antimicrobial resistance among Gram-negative bacteria in animals and their public health impact. Essays Biochem 2017;61(1):23-35. https://doi.org/10.1042/ebc20160055

19. J Le, Nguyen T, Okamoto M, et al. Impact of empiric antibiotic use on development of infection caused by extended-spectrum B-lactamase bacteria in a neonatal intensive care unit. Pediat Infect Dis J 2008;27(4):314-318. https://doi.org/10.1097/inf.0b013e3181606850

20. Allegranzi B, Luzzati R, Luzzani A, et al. Impact of antibiotic changes in empirical therapy on antimicrobial resistance in intensive care unit-acquired infections. J Hosp Infect 2002;52(2):136140. https://doi.org/10.1053/jhin.2002.1277

21. Van Duijn PJ, Verbrugghe W, Jorens PG, et al. The effects of antibiotic cycling and mixing on ntibiotic resistance in intensive care units: A cluster-randomised crossover trial Lancet Infect Dis 2018;18(4):401-409. https://doi.org/10.1016/s1473-3099(18)30056-2

22. Vincent J-L, Rello J, Marshall J, et al. International study of the prevalence and outcomes of infection in intensive care units. JAMA 2009;302(21):2323-2329. https://doi.org/10.1001 jama.2009.1754

23. Law T, Chibabhai V, Nana T. Analysis and comparison of cumulative antibiograms for the Charlotte Maxeke Johannesburg Academic Hospital adult intensive care and high-care units, 作

24. Ramsamy Y, Muckart DJJ, Han KSS. Microbiological surveillance and antimicrobial stewardship minimise the need for ultrabroad-spectrum combination therapy for treatment of nosocomial infections in a trauma intensive care unit: An audit of an evidence-based empiric antimicrobia policy. S Afr Med J 2013;103(6):371-376. https://doi.org/10.7196/samj.6459

25. Ramsamy Y, Essack S, Sartorius B et al. Antibiotic resistance trends of ESKAPE pathogens in Kwazulu-Natal, South Africa: A five-year retrospective analysis. Afr J Lab Med 2018:7(2):a887. https://doi.org/10.4102/ajlm.v7i2.887

26. Von Knorring N, Nana T, Chibabhai V. Cumulative antimicrobial susceptibility data for a tertiary-level paediatric oncology unit in Johannesburg, South Africa. S Afr J Onco 2019;3(0):1-9. https://doi.org/10.4102/sajo.v3i0.65

27. Kaufman D. Haas CE, Edinger R, et al. Antibiotic susceptibility in the surgical intensive care unit compared with the hospital-wide antibiogram. Arch Surg 1998;133(10):1041. https://do org/10.1001/archsurg.133.10.1041

28. Lowman W. Minimum inhibitory concentration-guided antimicrobial therapy - the Achilles heel in the antimicrobial stewardship agenda. S Afr Med J 2018;108(9):710. https://doi. org/10.7196/samj.2018.v108i9.13285

29. World Health Organization. Step-by-step approach for development and implementation of hospital and antibiotic policy and standard treatment guidelines. Geneva: WHO, 2011. https:// apps.who.int/iris/handle/10665/205912 (accessed 31 January 2020).

30. Chetty S, Reddy M, Ramsamy Y, et al. Antimicrobial stewardship in South Africa: A scopin review of the published literature. JAC Antimicrob Resist 2019;1(3):2632-1823. https://doi. org/10.1093/jacamr/dlz060

Accepted 2 July 2020 